\title{
Traditional Chinese Medicine in Neuroprotection after Brain Insults with Special Reference to Radioprotection
}

\author{
Xiao Chun Peng, ${ }^{1}$ Jiang Rong Huang, ${ }^{1}$ Si Wei Wang, ${ }^{1}$ Lian Liu, ${ }^{1}$ Zhen Zhen Liu, \\ Gautam Sethi $\mathbb{D},{ }^{2}$ Bo Xu Ren $\left(\mathbb{1},{ }^{1}\right.$ and Feng Ru Tang $\mathbb{1}^{3}$ \\ ${ }^{1}$ Medical School of Yangtze University, Jingzhou 434000, China \\ ${ }^{2}$ Department of Pharmacology, Yong Loo Lin School of Medicine, National University of Singapore, Singapore 117600 \\ ${ }^{3}$ Radiation Physiology Laboratory, Singapore Nuclear Research and Safety Initiative, National University of Singapore, \\ 1 CREATE Way \#04-01, CREATE Tower, Singapore 138602
}

Correspondence should be addressed to Bo Xu Ren; boxuren188@163.com and Feng Ru Tang; tangfr@gmail.com

Received 16 October 2017; Revised 23 January 2018; Accepted 18 November 2018; Published 26 November 2018

Academic Editor: Khalid Rahman

Copyright (C) 2018 Xiao Chun Peng et al. This is an open access article distributed under the Creative Commons Attribution License, which permits unrestricted use, distribution, and reproduction in any medium, provided the original work is properly cited.

\begin{abstract}
With rapidly increased construction of nuclear power plants worldwide to reduce energy shortage and subsequent environment contamination, routine use of radiotherapy and radiodiagnosis equipment in the clinical medicine, the research on the health effect of radiation exposure has become a very important area to explore. Traditional Chinese Medicine (TCM) may be an ideal candidate therapy as it usually produces fewer side effects even with long-term administration. In this paper, we reviewed current therapeutic approaches to prevent radiation-induced brain neuropathological and functional changes. Neuroprotective effects of TCM in different brain injury models have been briefly summarized. We then reviewed the neuroprotective and radioprotective effect of TCM in different radiation exposure models and discussed the potential molecular mechanism(s) of the neuroprotective and radioprotective effect of TCM. The conclusions and future research directions were made in the last part of the paper.
\end{abstract}

\section{Introduction}

Radiation-Induced Brain Dysfunction. Nuclear accidents such as radiation leakage from the Fukushima nuclear power plant in Japan in 2011, extensive use of X-ray, Computed Tomography (CT scan), Positron Emission Tomography (PET) in medical diagnosis, radiotherapy (RT) for treatment of human cancers, space travel, and atomic weapons testing have significantly increased the chance of radiation exposures [1]. Exposure to high doses/dose rates of radiation leads to an increased risk for cancer and noncancerous diseases including atherosclerotic, cardiovascular, cerebrovascular, and neurodegenerative effects [2]. Irradiation of eukaryotic cells induces damage to proteins, lipids, and DNA directly or indirectly due to free radical formation. Cell signaling events in response to radiation depend on environmental conditions besides genetic and physiological features of the biological systems [3]. In the mammalian brain, severe structural and functional injury occurs after acute or fractionated high dose radiation exposure [4]. Low doses/dose rates of radiation exposure may produce cognitive impairment even without any significant morphological alterations [5]. Ionizing radiation (IR) provokes cognitive deficits, especially during childhood and adolescence.

Different psychiatric disorders, including depression, bipolar disorder, and schizophrenia, may be related to hippocampal neurogenesis disturbances. There is evidence of an increased incidence of schizophrenia spectrum disorders following exposure to atomic bombing radiation, radiotherapy, or environment with high natural IR level [6]. Alzheimer's disease $(\mathrm{AD})$ is a human neurodegenerative disease, and its global prevalence has been predicted to increase dramatically in the following decades. Mounting evidence suggests that exposure to IR may result in the development of AD [7]. On the other hand, retrospective studies involving the general population and those with brain radiotherapy did not show any association between RT and Alzheimer's disease [8, 9]. From a therapeutic point of view, so far, only Amifostine 
has been used as an important adjunct to radiotherapy to reduce radiation-induced damage to normal tissues or cells, particularly in skin, intestine, marrow, mucosa, and salivary glands with lesser activity in kidney and lung and none in brain [10]. However, toxic side effects of Amifostine have restricted its use in clinical treatment of radiation-induced diseases [11]. Therefore, it is important to develop compounds which can protect against radiation-induced brain damage with less side-effect.

In the behavioral tests to evaluate the effect of Traditional Chinese Medicine (TCM) on learning and memory, Morris water maze test has been commonly used. The maze consists of a pool, with a hidden platform submerged just below the water surface. During the Morris water maze test, the rat or mouse learns to escape from the water by locating a hidden platform with the help of visual cues. The learning ability is quantified as escape time. The shorter escape time a mouse or a rat needed to find the central platform, the better its spatial memory [12].

In this review paper, a comprehensive literature research was carried out using key words "Traditional Chinese Medicine (TCM), radiation or irradiation, neuronal damage, brain, neuroprotection, or radioprotection" by means of the scientific engine Google Scholar (http://scholar.google.com/), and via the databases, PubMed (http://www.ncbi.nlm.nih .gov/pubmed). The following Chinese websites, http://acad .cnki.net/Kns55/brief/result.aspx?dbPrefix=CJFQ from China National Knowledge Infrastructure (CNKI), http://g.wanfangdata.com.cn/ from WanFang, and http://qikan.cqvip .com/ from WEIPU, were also searched.

\section{Current Treatment For Radiation-Induced Brain Dysfunction}

Radiation-induced neuronal apoptosis results from oxidative stress, and antioxidant treatments prevent radiation-induced brain damage [13]. Amifostine has been found to decrease the reactive oxygen species (ROS) levels [14] and suppress radiation-induced cell death in developing cerebellar granular cells [15]. Amifostine significantly attenuated recognition memory defects in adult mice exposed to low dose radiation [16] and has been widely used as a radioprotective agent.

Irradiation induces the activation of inflammatory cells and the release of inflammatory cytokines [17]. Antiinflammation therapy has been proved to be radioprotective. Eicosapentaenoic acid is an anti-inflammation agent and could effectively protect hippocampal neurons from damage by whole body irradiation [18, 19]. Pretreatment with anti-inflammatory drugs such as indomethacin or a peroxisome proliferator-activated receptor- $\alpha$ agonist combined with fenofibrate prevented microglial activation and impairment of neurogenesis [20]. Treatment with the angiotensin converting enzyme inhibitors AT1RA L-158,809 and ramipril ameliorated radiation-induced cognitive deficits and reduced apoptosis among subgranular zone (SGZ) progenitors and inflammatory disruption within the SGZ microenvironment in the rat model $[21,22]$. The administration of atorvastatin combined with ramipril appeared to synergistically ameliorate radiation-induced inhibition of neurogenesis [23].
Therefore, anti-inflammatory therapy may be a potential therapeutic approach for radiation-induced brain injury.

\section{Traditional Chinese Medicine (TCM) with Neuroprotective Effect}

Several TCM have been tested for their neuroprotective activity after brain insults (Table 1).

3.1. Morinda officinalis. Bajisin is a glycoside monomer extracted from TCM Morinda officinalis. It protects brain cells and has antiaging and antidepression effect. In the rat model of acute cerebral ischemic injury, Bajisin increased the activity of superoxide dismutase (SOD), glutathione peroxidase, and glucose production and reduced lipid peroxide in the brain tissue of senile mice. It had no obvious influence on the nitrogen monoxide (NO) [24]. In D-galactose and sodium nitrite-induced Alzheimer disease model, Morinda officinalis significantly increased the learning and memory ability [25]. It also had antiaging effect. Morinda officinalis significantly decreased malondialdehyde (MDA) and the apoptotic index of Purkinje fibers [26]. So far, five compounds from Morinda officinalis were isolated and their structures were identified as asrubiadin-1-methylether (I), 2-hydroxy1-methoxyanthraquinone (II), scopoletin (III), isofraxidin (IV), and anthraquinone-2-aldehyde (V) [27]. When the forced swimming tests in mice and rats and differentialreinforcement-of-low-rate 72 second schedule (DRL72 s) in rats were used, the extracts induced significant reduction in the immobility periods in the forced swimming tests and elicited significant increases in reinforcers in DRL72 s [28]. Clinical trials suggested that Bajitian oligosaccharide capsule improved the symptoms of patients with mild or moderate depression. The efficacy was similar to fluoxetine, but it produced fewer side effects $[29,30]$.

3.2. Lycium barbarum. Regulates immunity, has antiaging effect, and is able to scavenge free radicals. Administration of Lycium barbarum juice significantly improved learning and memory ability and increased the activities of acetylcholinesterase (AchE) and SOD, while the contents of MDA in brains decreased obviously when compared to aging mice [31]. It increased the learning and memory ability in manganese [32] or lead [33] poisoning mice model. Lycium barbarum could also induce differentiation of bone marrow stromal cells (BMSCs) into neurons [34].

3.3. Safflor. Improved the learning and memory ability of $\mathrm{AD}$ rats induced by AB1-42[35]. This effect may be related to the decrease of oxidative stress and the increase of cholinergic nerve function in brain tissue [36-38].

3.4. Epimedium. Is a genus of flowering plants in the family Berberidaceae. The active component of Epimedium extracts Icariin inhibits tumor, enhances immunity, improves heart and cerebral vessels, and regulates endocrine function. Recent studies suggest that Icariin also has neuroprotective effects in the central nervous system. Icariin increased the SOD activity of brain tissue, reduced MDA and AchE activity, 


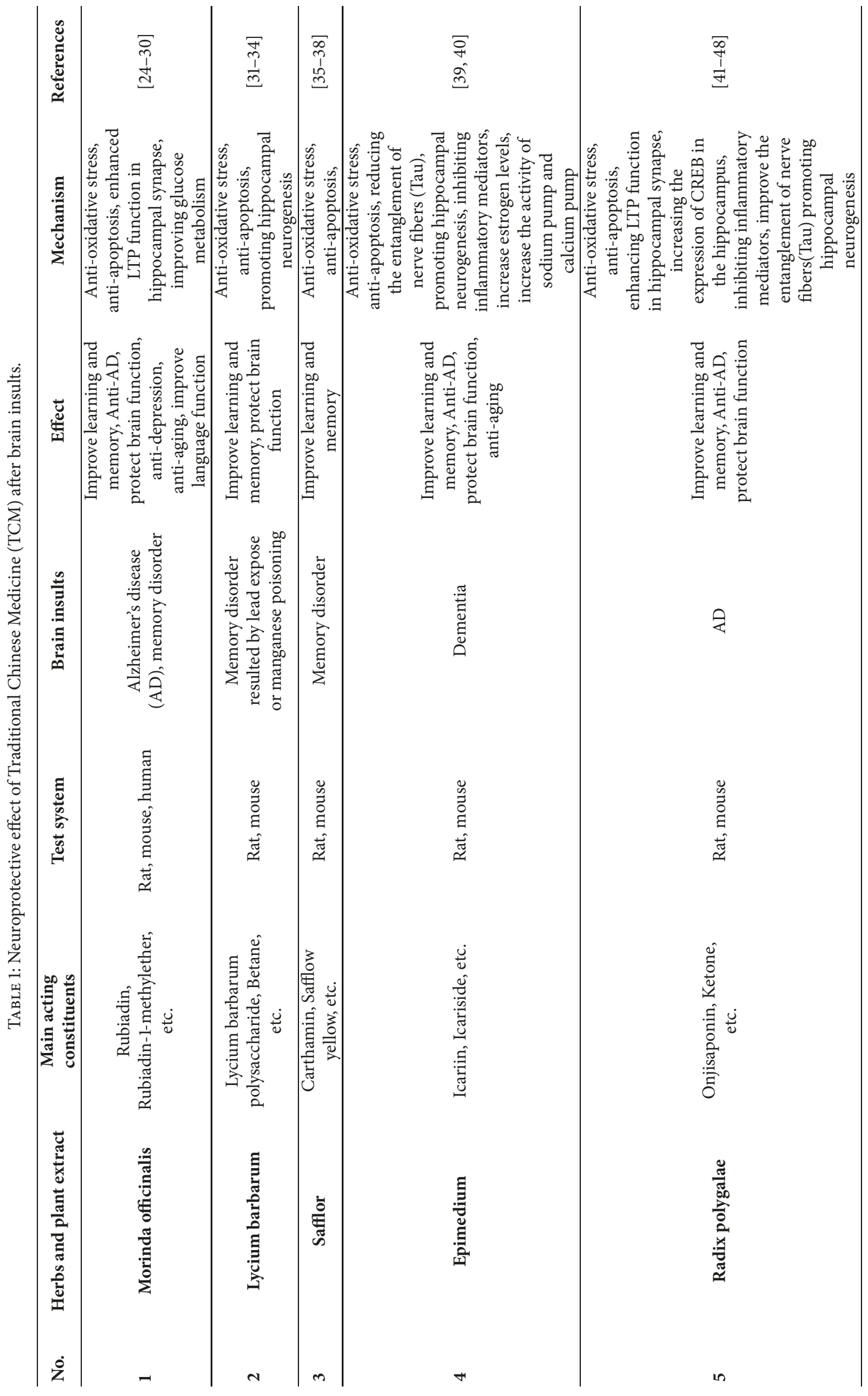




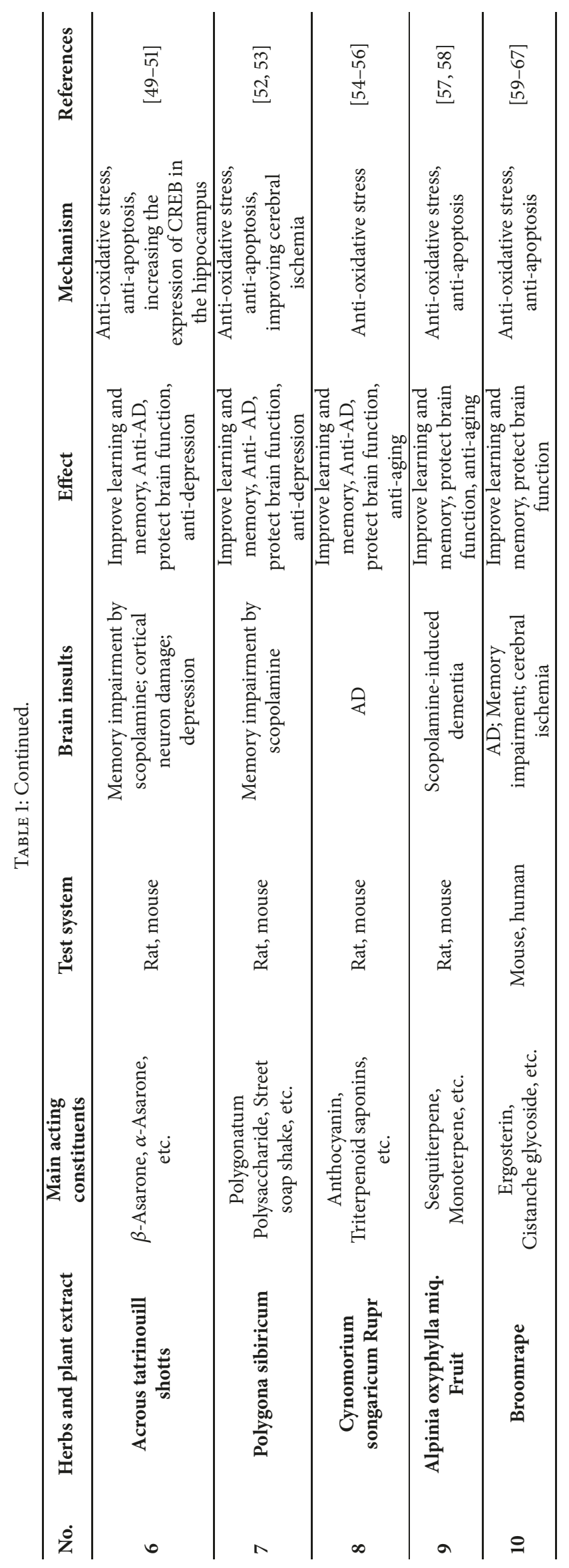


and therefore protected the hippocampus and improved the learning and memory ability in the rat model [39]. Icariin also promoted neurogenesis in the dentate gyrus (DG) of the hippocampus [40].

3.5. Radix polygalae. Is usually used for the treatment of human insomnia or coughing. In the rat model of Alzheimer's disease $(A D)$, Radix polygalae could effectively improve learning and memory ability by inhibiting the activity of brain AchE, reducing MDA, free radical levels, and oxidative stress injury, and increasing SOD [41]. It significantly decreased the escape latency in hidden platform and increased the time spent in target quadrant and the number of crossing times in the spatial probe test [42]. Radix polygalae also protected neurons from the toxic effect of AB1-40 and reduced the hyperphosphorylation of tau (Ser 396) in the neurons of $\mathrm{AD}$ rats by activating the expression of protein phosphatase 2A (PP2A) and inhibiting the expression of protein kinase A (PKA) [43]. The main active component of Radix polygalae, Tenuigenin, promoted the differentiation of neural stem cell into nerve cells $[44,45]$. Radix polygalae improved hippocampal-dependent learning and memory and had potential antidepressant properties [46-48].

3.6. Acrous tatrinouill shotts. Is widely used in clinical practice in epilepsy, fever, phlegm syncope, faint, forgetful, stroke aphasia, tinnitus, and Alzheimer's disease. B-asarone is its main ingredient. Acrous tatrinouill shotts can improve the learning and memory ability of rats induced by scopolamine, which may be linked to the reduction of the expression of glial fibrillary acidic protein (GFAP) and MDA in hippocampal astrocytes [49]. Glutamate exposure to cultured rat cortical neurons induced morphological changes and lactate dehydrogenase (LDH) leakage, increased intracellular calcium concentration, and decreased cell survival rate. B-asarone could reduce intracellular calcium concentration, LDH leakage, and apoptosis ratio and therefore increase cell survival [50]. The depression was relieved effectively by B-asarone in the rat model which may be related to the improvement of the expression of Bcl-2, brain-derived neurotrophic factor (BNDF), tyrosine kinase receptor $\mathrm{B}(\operatorname{TrkB})$, and mitogenactivated protein kinases (MAPK) [51].

3.7. Polygona sibiricum. Has antiaging, lowering increasing coronary blood flow, and anticancer effect. Polygona sibiricum improved the learning and memory ability in mice. Alcohol extract from the rhizome of Polygonatum sibiricum improved acquisition of impairment of memory induced by scopolamine (SCO) in mice. It also extended the survival time of mice subjected to cerebral ischemia by the occlusion of the bilateral carotid arteries and that PS ethanolic extract $2.0 \mathrm{mg} / \mathrm{mL}$ and $10.0 \mathrm{mg} / \mathrm{mL}$ inhibited MDA formation in the rat brain tissue [52]. Polygona sibiricum also reduced the deposition of $\mathrm{A} \beta$ in the hippocampus of $\mathrm{AD}$ rats [53].

3.8. Cynomorium songaricum Rupr. Has the role of tonifying kidney yang, enhancing aphrodisiac action, and laxative effect. It markedly improved the learning and memory ability in the rat $\mathrm{AD}$ model by reducing oxidative stress in the brain tissue and promoting the formation of synapses [54-56].

3.9. Alpinia oxyphylla miq. Is a dried ripe fruit of the perennial plant of the ginger family. It significantly improved the learning and memory ability in mice induced by scopolamine [57]. The Alpinia oxyphylla Miq could prevent the injury of hippocampal CA3 neurons in the restrained stress rat model [58].

3.10. Broomrape. Has different biological activities, such as immune regulation, memory enhancement, antioxidative, antiaging, and radiation protection. A significant improvement of memory was observed in mice with learning disabilities induced by hydrocortisone $[59,60]$. Broomrape protected the brain cells by scavenging oxygen free radicals and reducing lipid peroxidation damage to brain tissue $[61,62]$. It improved the learning and memory of AD mice by decreasing the content of MDA, increasing the activity of SOD, GSH$\mathrm{Px}$, and decreasing the activity of $\mathrm{AChE}$, the apoptosis rate of brain cells, and the accumulation of calcium in brain tissue [63-65]. In AD patients, Broomrape improved the cognitive and self-care ability and delayed the progress of dementia [66]. The total glycosides of Cistanche deserticola extract could significantly improve the behavioral characteristics in the mouse Parkinson's disease model induced by 1-Methyl-4phenyl-1,2,3,6-tetrahydropyridine (MPTP) and increase the content of dopamine in striatum and the expression of tyrosine hydroxylase in the substantia nigra [67].

\section{Traditional Chinese Medicine with Radioprotective Effects}

4.1. Astragalus membranaceus. Hydroponically grown root extracts from Astragalus membranaceus significantly reduced UVA-induced DNA damage in cultured human lung and skin fibroblasts [68]. In the brain of rats with acute encephalopathy caused by ${ }^{60} \mathrm{Co}$ irradiation, the intraperitoneal injection of Astragalus parenteral solution decreased the nitric oxide and ameliorated the cognitive ability, suggesting that astragale may protect radiation-induced brain injury $[69,70]$. The acute and chronic electromagnetic field (EMF) irradiation could initiate neurologic damage in hippocampus. Chinese medicine diet (CMD) which comprised ferulic acid, gimenoside, astragalus polysaccharide, an ingredient of Astragalus, and rhodiola sachaliens has protective effect on the impaired learning and memory, the neuronal apoptosis, and the peroxidation damage induced by electromagnetic field irradiation. CMD intervention played a significant protective role in antagonizing neurological damage in the later stage of acute irradiation and chronic irradiation $[71,72]$. Astragalus also significantly protected neuronal apoptosis induced by radiation injury at a single-dose X-ray exposure of $30 \mathrm{~Gy}$ [73]. While radiotherapy prolongs the survival time of patients with head and neck tumor, the side effects such as radiation optic neuropathy may lead to irreversible visual loss which seriously affects the quality of life of patients. Recent studies suggested that Astragalus membranaceus significantly improved the visual acuity of irradiated rats or 
patients with nasopharyngeal carcinoma after radiotherapy $[74,75]$.

4.2. Salvia miltiorrhiza. The bioactive constituents of Salvia miltiorrhiza, i.e., tanshinones and depsides, protect against $\beta$-amyloid-induced toxicity by the anti-inflammatory mechanisms. The two constituents enhance the antiapoptotic Bcell leukemia protein-2 family members, decrease the translocation of cytochrome $c$, and have an activity on vascular endothelial growth factor. In addition, depsides decrease caspase- 3 , intracellular $\mathrm{Ca}(2+)$, and reactive oxygen species while tanshinones enhance the activities of superoxide dismutase and glutathione peroxidase [76]. In the mouse whole brain irradiation model, Salvia miltiorrhiza prevented the high dose radiation-induced brain structural and functional changes and improved the quality of life by ameliorating the primary events [77]. Microwave irradiation induced a significant decrease of ATPase activity and a remarkable increase of $\mathrm{Na}^{+}, \mathrm{Ca}^{2+}$ contents in the hippocampus. However, Salvia miltiorrhiza could significantly lower the inhibition of ATPase activity and the increase of $\mathrm{Na}^{+}, \mathrm{Ca}^{2+}$ in the hippocampus. The neuronal damage was also ameliorated substantially [78]. The behavioral test indicated that Salvia miltiorrhiza could improve the learning and memory ability of rats [79]. This was confirmed by another study showing that Salvia miltiorrhiza improved the ionizing radiationinduced cognition impairment by reducing lipid peroxide (LPO) and intercellular cell adhesion molecule-1 (ICAM-1) expression in the mouse model [80]. The brain radioprotective effect of Salvia miltiorrhiza was also confirmed in clinical studies showing that radiotherapy combined with administration of Salvia miltiorrhiza significantly reduced radiation-induced brain injury [81-83].

4.3. Ligusticum chuanxiong Hort. Ligusticum chuanxiong Hort and its bioactive ingredient, tetramethylpyrazine (TMP), have been used to treat cardiovascular diseases and to relieve various neurological symptoms. TMP effectively protected neuronal apoptosis, which was associated with the inhibition of oxidative stress and a change in the levels of apoptosis-related proteins, Bcl-2 and Bax. Furthermore, TMP reduced the expression of proinflammatory cytokines such as TNF- $\alpha$ and IL-8, which likely contributes to its cytoprotective effects [84]. Clinical study has shown that TMP significantly improves the symptoms of patients with radiotherapy-induced encephalopathy [85] and optic neuropathy [86].

4.4. Broomrape. Citanche glycoside is an active component of Broomrape. It facilitated the repairing process of radiationinduced damage to the biological membranes and cells of sensitive organs in the mice [87].

4.5. Horse Chestnut P.E. $\beta$-aescine sodium is an ingredient from Horse Chestnut P.E. Cerebral edema is a radiation injury at acute stages after the exposure. Early aggressive treatment of cerebral edema could relieve the symptoms of intracranial hypertension, delay or block the development of the disease, and prevent the occurrence of cerebral hernia. Clinical data indicated that patients with radiation-induced brain edema could be effectively controlled by $\beta$-aescine sodium or mannitol and dexamethasone [88].

4.6. Radix Hedysari. In $\gamma$-ray irradiated rat model, administration of Radix hedysari significantly increased SOD, but decreased MDA activity in the brain tissue. It suggests that Radix hedysari may serve as an antioxidant drug and be helpful for the recovery of radiation-induced brain damage $[89,90]$. Radix hedysari capsule increased the wet weight of liver, spleen, brain, and testicular tissue of irradiated mice, suggesting that it has radioprotective effect [91].

4.7. Safflower. ${ }^{12} \mathrm{C}^{6+}$ irradiation induces cognitive dysfunction and impairment of the blood brain barrier, significantly decreased SOD, and increased MDA activity in the brain tissue. Hydroxysafflor yellow A, an ingredient of safflower, dose dependently improved cognitive dysfunction, protected the blood-brain barrier, increased SOD, and decreased MDA activity. It suggests that hydroxysafflor yellow A may have a radioprotective effect on radiation-induced brain injury [92].

4.8. Arnebiae Radix. Shikonin, a bioactive ingredient of Arnebiae Radix, improved ${ }^{12} \mathrm{C}^{6+}$ ion beam-induced brain injury by its modulating effects on the oxidative stress [93].

4.9. Ginkgo. Ginkgo flavonoid, an extract from Ginkgo, prevented age-related spatial memory deficits in both animal study [94] and clinical trial [95]. After high dose irradiation, Ginkgo flavonoid could prevent the radiation-induced hippocampal injury [96].

4.10. Ginseng. Panaxoside Rgl, a bioactive ingredient of Ginseng, reduced neuron apoptosis by controlling Cdk5 and played a protective role in radiation-induced hippocampal damage [97].

4.11. Kang-fu-ling. Kang-fu-ling (KFL) is a polybotanical dietary supplement with antioxidant properties. KFL reversed high power microwave-induced memory loss and the histopathological changes in hippocampus of rats. In addition, KFL displayed a protective effect against HPMinduced oxidative stress and activated the nuclear factor-E2related factor 2 (Nrf2) and its target genes in the hippocampus of rats. The Nrf2-antioxidant response element (ARE) signaling pathway may be involved in the neuroprotective effects of KFL against HPM-induced oxidative stress. The dietary supplement KFL may therefore be a promising natural radioprotector [98].

4.12. Shenqi. Shenqi Fuzheng Injection (SFI) was extracted from a number of medicinal herbs, such as Radix Codonopsis (root of Codonopsis pilosula) and Radix Astragali (root of Astragalus), and was approved by the State Food and Drug Administration of China in 1999. Administration of SFI effectively attenuates irradiation-induced brain injury via inhibition of the NF- $\kappa$ B signaling pathway and microglial activation [99].

4.13. 978-1. A TCM of destagnation and renal invigoration (978-1) was effective to prevent or treat the damage of learning 
and memory ability caused by irradiation in mice. It was able to prevent or treat radiation-induced subventricular cell damage by downregulation of p53 and C-jun expression and inhibition of apoptosis $[100,101]$.

\section{The Molecular Mechanisms of Neuroprotective and Radioprotective Effect of TCM}

It has been well documented that radiation induces brain oxidative stress, microglial activation, acute and chronic inflammatory responses, apoptosis, autophagy, abnormal angiogenesis and neurogenesis, redistribution or imbalanced neurotransmitter and receptor systems, and downregulation of neural growth factors [102]. TCM may play roles in antioxidative stress and antiapoptosis. It could promote hippocampal neurogenesis, improve microcirculation, inhibit microglial activation and inflammation, and reduce TAU production (Table 2).

The roles of antioxidative stress and antiapoptosis of TCM have been well accepted. Morinda officinalis increases the activity of superoxide dismutase (SOD) and glutathione peroxidase (GSH-Px) in the brain tissue of rats with acute cerebral ischemia and aging and reduce the content of lipid peroxide (LPO) [24, 26]. In the Lycium barbarum-treated aging mice induced by $\mathrm{D}$-galactose, the animal learning, memory, and brain AchE and SOD increased significantly, while brain MDA decreased obviously. Similar changes were also found in Lead poisoning mice [31,33]. Safflor increased SOD and GSH-Px, but decreased MDA in the cortical tissue of AD rats [35-38, 92]. Epimedium improved animal learning and memory ability by increasing brain SOD, decreasing MDA and Ach E, and reducing the damage of hippocampal neurons by $\mathrm{D}$-gal $\mathrm{AlCl}_{3}$ treatment [39]. In the $\mathrm{AD}$ model induced by the hippocampal injection of amyloid- $\beta 25 \sim 35$, the brain SOD were decreased, but AchE and MDA were increased obviously leading to learning and memory impairment. Radix polygalae treatment increased SOD, reduced AchE and MDA, and improved learning and memory ability [41]. Similarly, Acrous tatrinouill shots [49, 51, 103], Polygona sibiricum [52], and Cynomorium songaricum Rupr [54-56, 104, 105] also increased the brain SOD, reduced MDA, and improved the impairment of learning and memory, whereas Alpinia oxyphylla miq. fruit, Broomrape, and Astragalus membranaceus are antioxidative and antiapoptotic [57-75, 87], and Salvia miltiorrhiza decreased the brain lipid peroxidase in $\mathrm{AD}$ rats $[76,80,81]$. Ligusticum chuanxiong Hort inhibited free radicals in hypoxia and reduced neuronal apoptosis [85]. In ${ }^{60} \mathrm{Co}-$ $\gamma$ irradiated animals, Radix Hedysari treatment increased the brain superoxide dismutase activity but reduced maleic dialdehyde $[89,90]$. Other antioxidative stress and antiapoptosis TCM include Arnebiae Radix, Ginkgo, Ginseng, Kang-fu-ling, Shenqi, and Renal invigoration (978-1) [93-101].

TCM may also promote neurogenesis to prevent radiation-induced cognitive impairment. Morinda officinalis and Lycium barbarum promoted neurogenesis in the subgranular zone of the dentate gyrus $[29,32,34]$. Morinda officinalis also increased the number of dendrites and their branches of the hippocampal neurons [29], whereas Lycium barbarum had inductive effect on differentiation of bone marrow stromal cells (BMSCs) into neurons [32, 34]. Epimedium significantly reduced senile plaques in the hippocampus and increased the number of BrdU+ cells in the dentate gyrus. Our previous study showed that epimedium extract prevented the loss of proliferation cells, newly generated neurons, and interneurons in the hilus, in particular, the subgranular zone of the dentate gyrus $[40,106]$. In vitro study showed that adding tenuigenin to the neural stem cell medium increased the number of newly formed neurospheres and promoted the differentiation of the hippocampal neural stem cells (NSCs) into neurons [44-46, 48].

TCM improved radiation-induced inflammation and microcirculation changes. TMP reduced the expression of proinflammatory cytokines such as TNF- $\alpha$ and IL-8, which may also contribute to its cytoprotective effects [84]. Horse Chestnut P.E. improved microcirculation and stabilizing vascular endothelial cells and has been used to treat radiation-induced brain edema [88]. In rats with AB25-35 induced Alzheimer's disease, Epimedium was used to improve spatial learning and memory by inhibiting TNF-a, IL-6, and caspase-3 expression [107]. Radix polygalae reduced the brain tau level, the phosphorylation of tau protein, and the expression of PKA, but increased the expression of PP2A in amyloid $\beta$ peptide 1$40\left(\mathrm{~A} \beta_{1-40}\right)$-injected mice [43]. Salvia miltiorrhiza inhibited the radiation-induced senile plaques and neurofibrillary tangles in the mouse brain [77], whereas Shengqifuzheng effectively attenuated irradiation-induced brain injury by inhibiting NF- $\kappa \mathrm{B}$ signaling pathway and microglial activation [99].

TCM may increase estrogen level and change the $\mathrm{Ca}^{2+}$ $\mathrm{Na}^{+}$function to protect brain injury. Acrous tatrinouill shots could reduce intracellular calcium concentration [50]. The oral administration of icariin increased serum estradiol(E_2) level and improved the learning and memory ability in AD rats [108]. Salvia miltiorrhiza increased ATPase activity and reduced $\mathrm{Na}+\mathrm{Ca} 2+$ in the hippocampus (CA1 area) and improved microwave radiation-induced brain damage [78] (refer to Figure 1).

\section{Limitation of TCM as Radio-Neuro-Protectants}

While it seems promising to use TCM as potential radioneuro-protectants, it should be emphasized that the ingredients of TCM are very complex and have not been fully identified and purified. Improper processing, dispensing, compatibility, excessive dosage, and individual differences may significantly affect clinical use of TCM [109]. As individual reponse to a same TCM may be different, it may compromise the usage of TCM for treatment of victims with massive radiation exposure. TCM Formulae with two or more herbs often produce better curative efficacies and fewer side effects than a single herb, but improper use may produce more harm than benefit [110]. Disbelief of TCM may also limit its use worldwidely. 


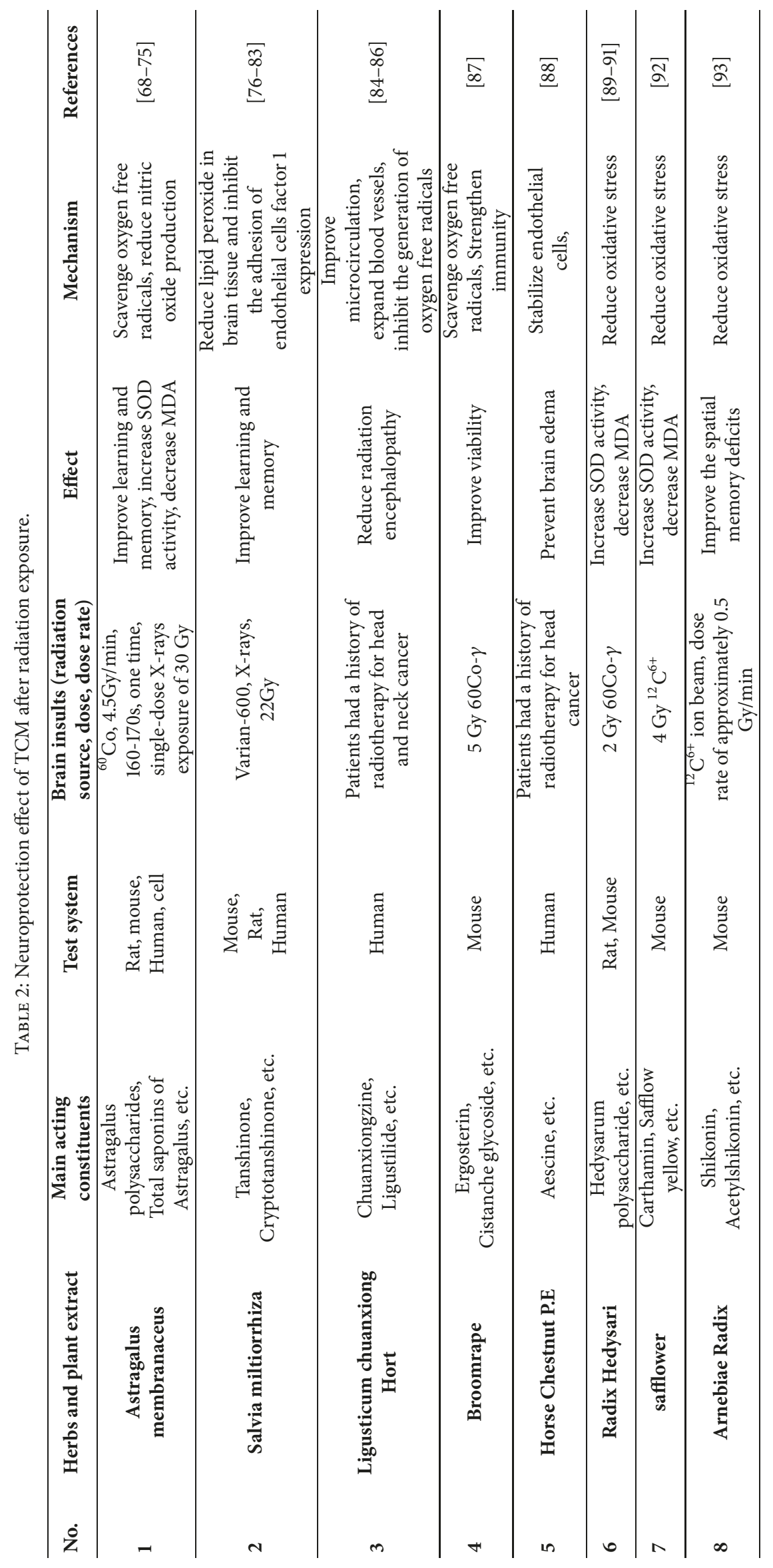




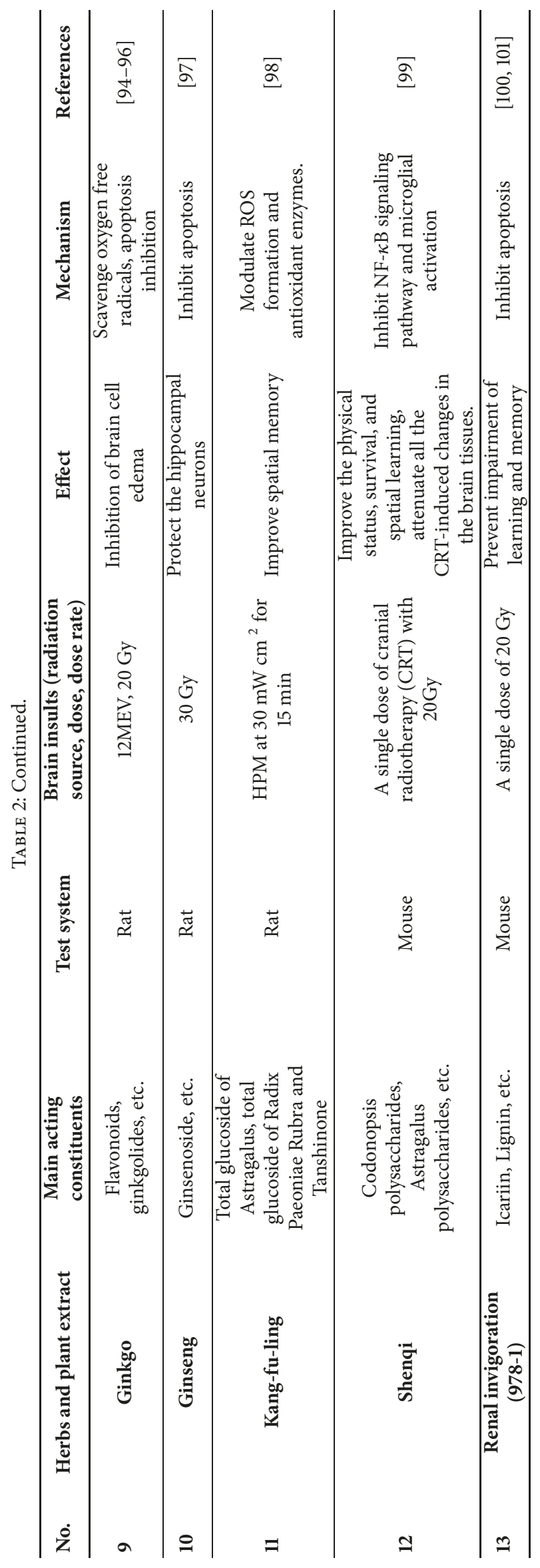




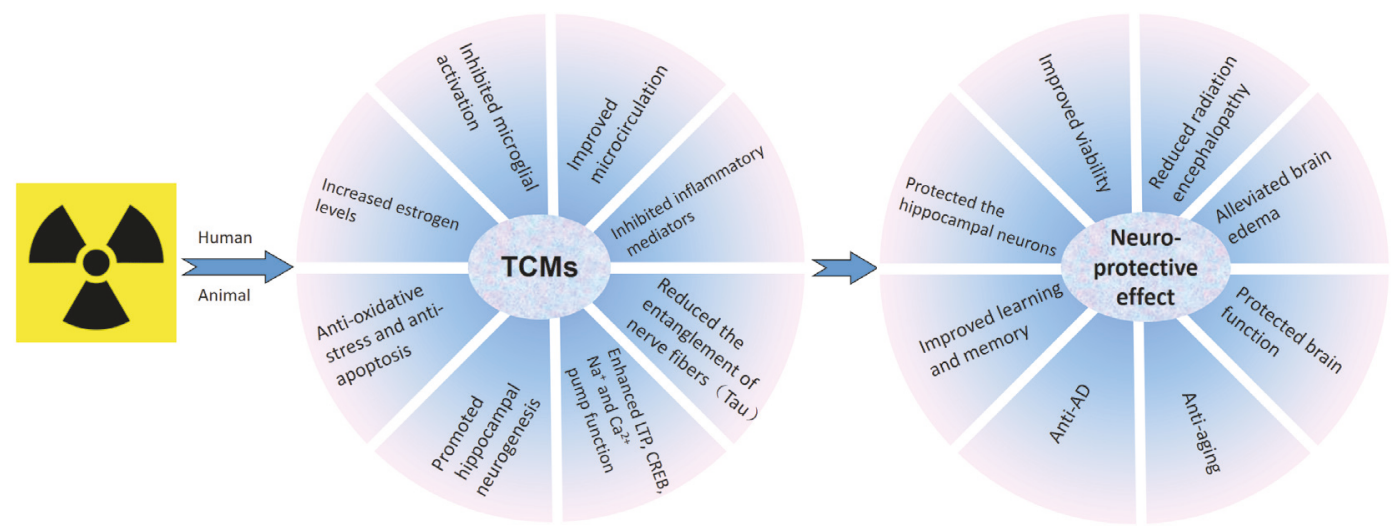

FIgURE 1: Traditional Chinese Medicine (TCM) reduces radiation-induced microglial activation and production of inflammatory cytokines and chemokines to inhibit brain inflammation. TCMs increase the superoxide dismutase activity and decrease malondialdehyde and nitric oxide production to reduce oxidative stress. Upregulation of Bcl-2, downregulation of Bax-2, downregulation of p53 and c-jun expression, and increased ratio of Bcl-2/Bax by TCMs prevent radiation-induced apoptosis. TCMs may also increase brain-derived neurotrophic factor (BNDF), tyrosine kinase receptor B (TrkB), and mitogen-activated protein kinases (MAPK) to promote neurogenesis and improve radiationinduced impairment of cognition. These drugs also enhance LTP, CREB, $\mathrm{Na}^{+}$and $\mathrm{Ca}^{2+}$ pump function, increase estrogen levels, and reduce the entanglement of nerve fibers (Tau) to improve radiation-induced neurocognitive impairment.

\section{Conclusions and Future Research Directions}

Extensive publications on neuroprotective and radioprotective effect of TCM suggest that TCM may be effective in prevention of radiation-induced glial cell activation and proliferation, neuroinflammation, oxidative stress, apoptosis, and neurodegeneration. TCM may also promote brain neurogenesis and improve radiation-induced impairment of cognition. However, different doses and combination of TCM, animal species, strains, ages, and sexes were used in different research laboratories in previous studies. The variations in radiation sources, doses/dose rates, and irradiation patterns (acute or fractionated) made it difficult to evaluate if the positive effect of TCM in one animal model or laboratory could be applied to other models or laboratories. The neuroprotective or radioprotective effect of TCM administered before irradiation may not be observed when TCM are injected after irradiation. The routes of TCM administration, i.e., oral or intraperitoneal injection, may also compromise the data translation. Furthermore, the purity of the components and composition of the compounds of TCM were not clearly mentioned in most of the previous studies which may limit its clinical use.

TCM with radioprotective effect on the brain are far less investigated than on other organs. Further extensive studies in the following areas may still be needed: (1) Radiosensitivity varies significantly among different strains of animals. The effect of TCM may have to be tested in the same strain of animals for comparison in order to make solid conclusions. Animal age and sex should be chosen carefully as TCM effect may be age- and sex-dependent. (2) When animals are exposed to the same dose of radiation, radiation dose rate may affect radiosensitivity. Therefore, the same dose rate of radiation exposure should be used to compare TCM effect. The radiation source and component of radiation may also affect TCM effect. (3) The mechanism of high dose/dose rate radiation-induced brain damage may be different from low dose/dose rate radiation-induced injury. Comparative study of radioprotective effect of TCM in animals exposed to high and low doses of radiation exposures, in particular, the latter, may shed light on further understanding the mechanisms of the two patterns of radiation-induced brain damage.

\section{Conflicts of Interest}

The authors have no actual or potential conflicts of interest.

\section{Authors' Contributions}

Xiao Chun Peng and Jiang Rong Huang contributed equally to this work.

\section{Acknowledgments}

This work was supported by Sciences Foundation of the Hubei Provincial Department of Education (Q20161305), Hubei Province Health and Family Planning Scientific Research Project (WJ2016-Y-10) to HJR, and National Research Foundation (NRF) of Singapore to TFR.

\section{References}

[1] J. S. Kim, M. Yang, S. H. Kim, T. Shin, and C. Moon, "Neurobiological toxicity of radiation in hippocampal cells," Histology and Histopathology, vol. 28, no. 1, pp. 301-310, 2013.

[2] D. Marazziti, S. Baroni, M. Catena-Dell'Osso et al., "Cognitive, psychological and psychiatric effects of ionizing radiation exposure," Current Medicinal Chemistry, vol. 19, no. 12, pp. 18641869, 2012.

[3] United Nations Scientific Committee on the Effects of Atomic Radiation, Sources and Effects of Ionizing Radiation, United Nations Scientific Committee on the Effects of Atomic Radiation, 2008.

[4] P. J. Tofilon and J. R. Fike, "The radioresponse of the central nervous system: A dynamic process," Journal of Radiation Research, vol. 153, no. 4, pp. 357-370, 2000. 
[5] J. S. Kim, H. J. Lee, J. C. Kim et al., "Transient impairment of hippocampus-dependent learning and memory in relatively low-dose of acute radiationsyndrome is associated with inhibition of hippocampal neurogenesis," Journal of Radiation Research, vol. 49, no. 5, pp. 517-526, 2008.

[6] K. N. Loganovsky, S. V. Volovik, K. G. Manton, D. A. Bazyka, and P. Flor-Henry, "Whether ionizing radiation is a risk factor for schizophrenia spectrum disorders?" The World Journal of Biological Psychiatry, vol. 6, no. 4, pp. 212-230, 2005.

[7] N. Begum, B. Wang, M. Mori, and G. Vares, "Does ionizing radiation influence Alzheimer's disease risk?" Journal of Radiation Research, vol. 53, no. 6, pp. 815-822, 2012.

[8] L. M. DeAngelis, J.-Y. Delattre, and J. B. Posner, "Radiationinduced dementia in patients cured of brain metastases," Neurology, vol. 39, no. 6, pp. 789-796, 1989.

[9] M. Peper, S. Steinvorth, P. Schraube et al., "Neurobehavioral toxicity of total body irradiation: A follow-up in long- term survivors," International Journal of Radiation Oncology Biology Physics, vol. 46, no. 2, pp. 303-311, 2000.

[10] T. L. Phillips, "Rationale for initial clinical trials and future development of radioprotectors," American Journal of Clinical Oncology, vol. 3, no. 2, pp. 165-173, 1980.

[11] G. C. Jagetia, "Radioprotective potential of plants and herbs against the effects of ionizing radiation," Journal of Clinical Biochemistry and Nutrition, vol. 40, no. 2, pp. 74-81, 2007.

[12] J. Li, X. Peng, X. Zeng et al., "Estrogen secreted by mesenchymal stem cells necessarily determines their feasibility of therapeutical application," Scientific Reports, vol. 5, no. 1, 2015.

[13] E. Sleeper, C. Tamm, J. Frisén, B. Zhivotovsky, S. Orrenius, and S. Ceccatelli, "Cell death in adult neural stem cells," Cell Death \& Differentiation, vol. 9, no. 12, pp. 1377-1378, 2002.

[14] M. Yang, M. S. Song, S. H. Kim et al., "Cytotoxicity of gamma-ray in rat immature hippocampal neurons," Journal of Veterinary Science, vol. 12, no. 3, pp. 203-207, 2011.

[15] L. R. Guelman, J. I. Cabana, R. M. Pagotto, and L. M. Zieher, "Ionizing radiation-induced damage on developing cerebellar granule cells cultures can be prevented by an early amifostine post-treatment," International Journal of Developmental Neuroscience, vol. 23, no. 1, pp. 1-7, 2005.

[16] H. Lee, J. Kim, M. Song et al., "Amifostine ameliorates recognition memory defect in acute radiation syndrome caused by relatively low-dose of gamma radiation," Journal of Veterinary Science, vol. 11, no. 1, pp. 81-83, 2010.

[17] C. Feiock, M. Yagi, A. Maidman, A. Rendahl, S. Hui, and D. Seelig, "Central nervous system injury - A newly observed bystander effect of radiation," PLoS ONE, vol. 11, no. 9, 2016.

[18] T. Babcock, W. S. Helton, and N. J. Espat, "Eicosapentaenoic acid (EPA): An antiinflammatory $\omega-3$ fat with potential clinical applications," Nutrition Journal, vol. 16, no. 11-12, pp. 1116-1118, 2000.

[19] P. E. Lonergan, D. S. D. Martin, D. F. Horrobin, and M. A. Lynch, "Neuroprotective effect of eicosapentaenoic acid in hippocampus of rats exposed to $\gamma$-irradiation," The Journal of Biological Chemistry, vol. 277, no. 23, pp. 20804-20811, 2002.

[20] S. Ramanan, M. Kooshki, W. Zhao, F. Hsu, D. R. Riddle, and M. E. Robbins, "The PPAR $\alpha$ agonist fenofibrate preserves hippocampal neurogenesis and inhibits microglial activation after whole-brain irradiation," International Journal of Radiation Oncology Biology Physics, vol. 75, no. 3, pp. 870-877, 2009.
[21] K. A. Jenrow, S. L. Brown, J. Liu, A. Kolozsvary, K. Lapanowski, and J. H. Kim, "Ramipril mitigates radiation-induced impairment of neurogenesis in the rat dentate gyrus," Journal of Radiation Oncology, vol. 5, no. 1, article no. 6, 2010.

[22] T. C. Lee, D. Greene-Schloesser, V. Payne et al., "Chronic administration of the angiotensin-converting enzyme inhibitor, ramipril, prevents fractionated whole-brain irradiationinduced perirhinal cortex-dependent cognitive impairment," Journal of Radiation Research, vol. 178, no. 1, pp. 45-56, 2012.

[23] K. A. Jenrow, J. Liu, S. L. Brown, A. Kolozsvary, K. Lapanowski, and J. H. Kim, "Combined atorvastatin and ramipril mitigate radiation-induced impairment of dentate gyrus neurogenesis," Journal of Neuro-Oncology, vol. 101, no. 3, pp. 449-456, 2011.

[24] Z. F. Chen, B. X. Tan, J. W. Chen, X. Y. Li, R. S. Lin, and L. Lin, "Mechanism of Bajisins protective effect on acute cerebral ischemic injury," Journal of Guangzhou University Of TCM, vol. 17, no. 3, pp. 215-217, 2000.

[25] X. Wang, J. Li, Y. L. Liao, and C. L. Deng, "Study on protective eeffct of water extract from morindae officinalis on alzheimer disease model rats," China Pharmacy, vol. 24, no. 31, pp. 29082910, 2013.

[26] Z. Chen, R. F. Fu, and L. X. Cheng, "Effect of BEM on cerebellum of D-galactose induced aged rat," Journal of Chinese Medicine, vol. 25, no. 150, pp. 903-907, 2010.

[27] Y. B. Li, L. L. Wang, X. P. Lai, F. Feng, and Y. J. Zhou, "Active anti-aging constituents in Morinda officinalis," Central South Pharmacy, vol. 9, no. 2, pp. 101-103, 2011.

[28] Z. Q. Zhang, L. Yuan, N. Zhao, Y. K. Xu, M. Yang, and Z. P. Luo, "Antidepressant effect of the ehanolic extracts of the roots of Morinda officinalis in rats and mice," Chinese Pharmaceutical Journal, vol. 35, no. 11, pp. 739-741, 2000.

[29] L.-Y. Zou and H.-Y. Zhang, "Research advance of morinda officinalis oligosaccharides in treatment of depression," Chinese Journal of New Drugs, vol. 21, no. 16, pp. 1889-1945, 2012.

[30] T. L. Zhang, J. Liu, Halia. Bahati, B. Y. Li, and W. Y. Liu, "The value of CT perfusion and functional magnetic resonance imaging in the treatment of vascular dementia Morinda officinalis oligosaccharides Bajijiasu treatment of vascular dementia with short-term and long-term effect," Chinese Journal of Gerontology, vol. 36, no. 19, pp. 4765-4767, 2016.

[31] J. J. Ji, L. S. Han, J. Xu, Y. Yang, C. Y. Xu, and Y. Yan, "Effects of fructus lycii on learning and memory of D-galactose induced aging mice," Chinese Journal of Public Health, vol. 23, no. 3, pp. 359-360, 2007.

[32] J. Wen, B. N. Yang, and D. Ren, "Effects of Lycium barbarum polysaccharides on neurogenesis and learning and memory in manganese poisoning mice," Chinese Journal of Integrated Traditional and Western Medicine, vol. 30, no. 3, pp. 295-298, 2010.

[33] T. G. Cai, Y. Cai, and S. L. Yu, "Influence of Lycium barbarum polysaccharides on neurogenesis and learning and memory and protein kinase C in hippocamous in lead exposed mice," Chinese Journal of Public Health, vol. 26, no. 1, pp. 38-39, 2010.

[34] X. Liu, W. Shan, R.-X. Zeng, Y. Fang, D.-H. Li, and S.-J. Qin, "Differentiation of rat bone marrow mesenchymal stem cells into neuron-like cells induced by lycium barbarum polysaccharide," Journal of Clinical Rehabilitative Tissue Engineering Research, vol. 13, no. 14, pp. 2667-2672, 2009.

[35] Q. Ma, H. Xu, Y. Y. Ruang, X. M. Shi, Z. X. Wang, and Y. L. Hu, "Effects of safflower yellower on learning and memory disorders of dementia rats induced by AB1-42," Pharmacology and Clinics of Chinese Materia Medica, vol. 30, no. 5, pp. 64-66, 2014. 
[36] Y. L. Hu, P. L. Wang, R. Zhang, and M. Liu, "Effects of safflower yellow pigment on learning and memory in mice induced by scopolamine," Chinese Journal of Gerontology, vol. 10, no. 32, pp. 4197-4198, 2012.

[37] H. Xu, Q. Ma, Z. X. Wang, and Y. L. Hu, "Effects of safflower yellow on D-galactose/sodium nitrite induced learning and memory disorders in mice," Pharmacology and Clinics of Materia Medica, vol. 29, no. 2, pp. 59-61, 2013.

[38] H. Xu, Q. Ma, Z. X. Wang, and Y. L. Hu, "Effects of safflower yellow on learning and memory impairments of vascular dementia in rats in mice," Chinese Pharmacentical Journal, vol. 49, no. 12, pp. 1032-1035, 2014.

[39] W. Deng, M. G. Zheng, J. Zhang, and C. Huang, "Effects of total flavonoids from two species of the central Guizhou on the memory of rats with dementia," Lishizhen Medicine and Materia Medica Research, vol. 23, pp. 627-629, 2012.

[40] R. Cai, F. Li, H. X. Dong, and J. S. Shi, "Effects of icariin on the learning and memory function and hippocampus neurogenesis of Tg2576 mice," Journal of Zunyi Medical University, vol. 38, no. 4, pp. 350-354, 2015.

[41] H. T. Zhan, H. Y. Li, X. X. Zeng, and J. F. Wu, "Effects of mechanism of polygala hongkongensis hemsl extracts on learning and memory in Alzheimer's disease model rats," Journal of Apoplexy and Nervous Diseases, vol. 29, no. 3, pp. 243-245, 2012.

[42] C. R. Guo, "Effects of Radix polygalae by different processing methods on learning memory in model mice induce by scopolamine," Shandong Chemical Industry, vol. 44, no. 4, pp. 10-12, 2015.

[43] K. L. Xu, Q. Chen, W. Liu et al., "Effects of tenuigenin on tau protein phosphory lation at Ser 396 site in neurons of AD rats induced by AB1-40," Chinese Journal of Pathophysiology, vol. 28, no. 9, pp. 1605-1609, 2012.

[44] L. Huang and S. Y. Kuang, "Effects of volatile oil extracted from Alipinia oxyphylla on the apoptosis of substantial nigra neuron in PD mice model," China Pharmacy, vol. 22, no. 47, pp. 44304433, 2011.

[45] Y. Chen, X. Huang, W. Chen, N. Wang, and L. Li, “Tenuigenin promotes proliferation and differentiation of hippocampal neural stem cells," Neurochemical Research, vol. 37, no. 4, pp. 771777, 2012.

[46] W. Xue, J.-F. Hu, Y.-H. Yuan et al., "Polygalasaponin XXXII from polygala tenuifolia root improves hippocampal-dependent learning and memory," Acta Pharmacologica Sinica, vol. 30, no. 9, pp. 1211-1219, 2009.

[47] Y. Hu, P. Liu, D.-H. Guo, K. Rahman, D.-X. Wang, and T.-T. Xie, "Antidepressant effects of the extract YZ-50 from Polygala tenuifolia in chronic mild stress treated rats and its possible mechanisms," Pharmaceutical Biology, vol. 48, no. 7, pp. 794800, 2010.

[48] P. Liu, Y. Hu, D.-H. Guo et al., "Potential antidepressant properties of Radix Polygalae (Yuan Zhi)," Phytomedicine, vol. 17, no. 10, pp. 794-799, 2010.

[49] X. J. Zhou, S. J. Dai, H. S. Chen, F. R. Xie, C. Y. Li, and Y. X. Yang, "Effects of acorus tatarinowii schott volatile oil on learning and memory impairment rats induced by scopolamine and its possible mechanism," Journal of Gansu College of TCM, vol. 32, no. 1, pp. 1-6, 2015.

[50] Y.-Z. Chen, Q.-W. Wang, Y. Liang, and Y.-Q. Fang, "Protective effects of beta-asarone on cultured rat cortical neurons damage induced by glutamate," Journal of Chinese Medicinal Materials, vol. 30, no. 4, pp. 436-439, 2007.
[51] Y. R. Sun, H. Y. Dong, X. Y. Wang et al., "Influences of B -asarone on Rat behaviors and expression of hippo campal neurons in rats with depression," Journal of Beijing University of Traditional Chinese Medicine, vol. 36, no. 8, pp. 546-549, 2013.

[52] L. R. Sun, X. Li, Y. Y. Guo, D. Wang, and Z. Li, "A study on the effects of the rhizome of pokygonatum sibiricum red on acquisition of memory impairment induced by scopolamine in mice and the others," Journal of Shenyang Pharmaceutical University, vol. 18, pp. 286-289, 2001.

[53] Y. L. Yi, S. X. Wu, M. S. Ye, Y. Zeng, P. Zhang, and Y. Q. Xie, "Effect of A $\beta$ 1-42 injection on hippocampus cells in rats and protective role of polygona-polysaccharose for Alzheimer's disease," Journal of Central South University (Medical Sciences), vol. 39, pp. 344-348, 2014.

[54] Y.-Q. Zhao, Z.-W. Wang, and Y.-H. Jing, "The study on the effect of Suoyang on ultrastructural in the relevant brain areas of learning and memory in the rat's model of Alzheimer' disease," Chinese Journal of Clinical Rehabilitation, vol. 6, no. 15, pp. 22202221, 2002.

[55] F. Z. Tian, H. Y. Chang, J. Y. Zhou, and Y. Lu, "Effects of ethyl acetate extract from cynomorim songaricum on learning and memory function and hippocampa neurons in ovariectomized rat model with Alzheimer's disease," Journal of Beijing University of Traditional Chinese Medicine, vol. 37, no. 11, pp. 763-766, 2014.

[56] M. Wu, L. Liu, Y. L. Hu, and J. L. Liu, "Effect of water extracts of Cynomorium songaricum on scopolamine induced learning and memory impairment of mice," Xinjiang Journal of Traditional Chinese Medicine, vol. 33, no. 2, pp. 29-31, 2015.

[57] Y. Q. Li, Y. F. Wang, X. L. Shi, T. Y. Li, and Y. Zhang, "Effect of Ethanol extract of fruit of Alpinia oxyphylla Miq on learning and memory ability in scopolamine-induced dementia mice," Journal of Capital Normal University, vol. 36, no. 1, pp. 54-56, 2015.

[58] L. Sun, Y. J. Chen, C. Liu, and Y. Zhou, “The FAO may have an effect on the injury of hippocampal CA3 neurons in restrained stress rat," Journal of Dalian University of Technology, vol. 6, pp. 87-90, 2009.

[59] Y. M. Hu, Y. X. Hu, X. Y. Liu, Z. Ma, and C. Z. Yi, "Effects of total glycosides of Cistanche deserticola on learning and memory function of normal mice," Chinese Journal of Preventive Medicine, vol. 8, no. 4, pp. 370-373, 2007.

[60] C. Gao, C. S. Wang, G. Z. Wu, and P. F. Tu, "Effects of total glycosides of Cistanche deserticola on learning and memory function of mice with kidney yang deficiency induced by hydrocortisone," Chinese Journal of Traditional Chinese Medicine, vol. 11, no. 5, pp. 330-332, 2005.

[61] X. P. Pu, X. R. Li, H. N. Li, Z. F. Tu, Z. H. Song, and C. L. Li, "Protective effect of Cistanche deserticola campneoside on apoptosis induced by neurotoxin MPP+," Journal of Peking University (Health Sciences), vol. 33, no. 3, p. 217, 2001.

[62] X. Z. Meng, X. W. Wang, X. Y. Jiang, F. X. Liu, and H. T. Paer, "Protective effects of total glycosides of Cistanche deserticola on cerebral ischemia reperfusion injury in conscious mice," Chinese Journal of Clinical Neuroscience, vol. 11, no. 3, p. 239, 2003.

[63] L. Luo, X. W. Wang, F. X. Liu, S. Yang, and T. Wang, "The protective effect of meat Chengdu glycosides on aluminium trichloride induced learning and memory impairment of mice," Chinese Journal of New Drugs and Clinical Remedies, vol. 26, no. 1, pp. 33-36, 2007.

[64] F. X. Liu, X. W. Wang, X. F. Wang, and L. Luo, "Effect of glycosides of cistanche on learning and memory in Alzheimer's 
disease mice and its mechanism," Xinjiang Medical University, vol. 28, no. 12, pp. 1131-1135, 2005.

[65] F. X. Liu, X. W. Wang, L. Luo, H. Xin, and X. F. Wang, "Effects and mechanisms of total glycosides of Cistanche deserticola on learning and memory in mice with Alzheimer's disease caused by B - amyloid peptide," Chinese Pharmacological Bulletin, vol. 22, no. 5, pp. 595-599, 2006.

[66] Q. Wang, "The aclinicl research of Roucongrong Zonggan Jiaonang in treatment of Alzheimer's disease," Strait Pharmaceutical Journal, vol. 21, no. 3, pp. 103-104, 2009.

[67] X. C. Geng, L. W. Song, X. P. Pu, and P. Tu, "Neuroprotective effects of phenylethanoid glycosides from Cistanehes salsa agalnstl-methyl-4-phenyl-1, 2, 3, 6-tetrahydropyridine(MPIP)induced dopaminergic toxicity in C57 mice," Biological \& Pharmaceutical Bulletin, vol. 27, no. 6, pp. 797-801, 2004.

[68] A. Curnow and S. J. Owen, "An evaluation of root phytochemicals derived from althea officinalis (Marshmallow) and astragalus membranaceus as potential natural components of UV protecting dermatological formulations," Oxidative Medicine and Cellular Longevity, vol. 2016, Article ID 7053897, 9 pages, 2016.

[69] Y.-C. Xiao and Y. Chen, "Interventional effect of astragale parenteral solution on the cognitive ability and behavioral changes of rats with acute radioactive injury," Chinese Journal of Clinical Rehabilitation, vol. 9, no. 36, pp. 71-73, 2005.

[70] C. Z. Bai, Q. M. Zhong, Y. P. Wu, L. L. Jia, Y. Y. Niu, and M. L. Feng, "Experimental study on the protective effects of 5 kinds of Chinese herbs such as Astragalus membranaceus on radiation injury in mice," Chinese Journal of Cellular and Molecular Immunology, vol. 29, no. 10, pp. 1052-1054, 2013.

[71] Q. F. Gong, X. S. Yang, C. H. Deng, G. B. Zhang, and P. $\mathrm{Yu}$, "Protective effect of nutritional intervention on microwave induced oxidative damage in hippocampus of rats," Chinese Journal of Radiology Health, vol. 17, no. 4, pp. 401-403, 2008.

[72] Q. F. Gong, X. S. Yang, L. Tu, G. B. Zhang, and Z. P. Yu, “The chinese medicine nutrient diet intervention prevent against the neurologic damage induce by EMF irradiation in rat hippocampus," Chinese Journal of Applied Physiology, vol. 29, no. 4, pp. 346-350, 2013.

[73] H. H. Tang, Y. Chen, and J. Luo, "Protective effect of astragalus injection on radiation injury inprimary cultured rat hippocampal neuron," Chinese Journal of Cancer Prevention and Treatment, vol. 17, no. 14, pp. 1049-1051, 2010.

[74] D. Zhu, P. Zeng, J. Liu, L. Sun, and Y. T. Huang, "Traditional Chinese medicine for the treatment of radiation-induced optic neuropathy," Hubei Journal of Traditional Chinese Medicine, vol. 30, no. 4, pp. 39-40, 2008 (Chinese).

[75] J. P. Ji, X. H. Cheng, L. H. Lian, H. Liu, L. Chen, and Z. Y. Li, "Tongqiao stasis method on retinal vascular endothelial and optic nerve injury in rats and the preventive effect of radiation," Journal of Guangzhou University of Traditional Chinese Medicine, vol. 32, no. 4, pp. 715-719, 2015.

[76] L. Bonaccini, A. Karioti, M. C. Bergonzi, and A. R. Bilia, "Effects of salvia miltiorrhiza on CNS neuronal injury and degeneration: a plausible complementary role of tanshinones and depsides," Planta Medica, vol. 81, no. 12-13, pp. 1003-1016, 2015.

[77] J. L. Yi, Y. J. Liu, Y. J. Miao, W. Z. Yang, and W. M Cai, "Radiationinduced late brain injury and the protective effect of traditional chinese medicine," Chinese Journal of Radiation Oncology, vol. 13, no. 2, pp. 107-110, 2004.

[78] J. N. Zhang, X. Zhang, S. Y. Yi, and A. M. Li, "Neuronal damage in hippocampus( CA-1) region after microwave radiation with and without salvia miltiorrhiza treatment," Chinese Neurosurgical Journal, vol. 15, no. 1, pp. 18-20, 1999.

[79] S. J. Jiang, W. P. Wu, X. S. Zhang et al., "The improvement of Salvia miltiorrhiza on temporal lobe ischemia spatial cognitive impairment rats and on the expression of platelet-derived growth factor influence," Chinese Neurosurgical Journal, vol. 32, no. 1, pp. 290-292, 1999.

[80] J. L. Yi, Y. J. Liu, W. Z. Yang, X. D. Huang, Y. J. Miao, and W. M. Cai, "Radiation-induced acute brain injury and the protective effect of traditional chinese medicine-Salvia Miltiorrhiza," Chinese Journal of Radiation Oncology, vol. 12, no. 2, pp. 112-115, 2003.

[81] J. G. Mou, H. T. Zheng, and C. J. Zhang, "Effect of compound salvia miltiorrhiza injection on prevention of acute radiationinduced brain injury," Sichuan Tumor Prevention, vol. 17, no. 3, pp. 181-182, 2004.

[82] Y. L. Duan, X. H. Fan, Q. G. Liu, C. Zhao, and J. Guan, "Clinical observation of tanshinone IIA combined with radiotherapy in the treatment of elderly patients with brain metastases," Chinese Journal of Practical Medicine, vol. 38, no. 2, pp. 101-102, 2011.

[83] Y. L. Duan, X. H. Fan, Q. G. Liu, C. Zhao, and J. Guan, "Clinical observation on Tanshinone IIA in radiation induced senile brain injury," Modern Oncology, vol. 19, no. 4, pp. 674-675, 2011.

[84] C. Zhang, F. Teng, J. Tu, D. Zhang, and L. Annunziato, "Ultrasound-enhanced protective effect of tetramethylpyrazine against cerebral ischemia/reperfusion injury," PLoS ONE, vol. 9, no. 11, Article ID el13673, 2014.

[85] F. L. Yang, D. P. Luo, and Y. H. Tong, "Salviae miltiorrhizae liguspyragine hydrochloride and glucose injection treatment of radiation encephalopathy randomized controlled study," Chinese Journal of Clinical Research, vol. 27, no. 3, pp. 285-287, 2014.

[86] X. H. Chen, Z. Y. Li, and J. P. Ji, "Changes of visual vield and VEP in patients of radiation-induced optic neuropathy treated with ligustrazine combined with xingnaojing injection," Chinese Journal of Experimental Traditional Medical Formulae, vol. 19, no. 5, pp. 296-299, 2013.

[87] X. Y. Jiang, X. W. Wang, X. Y. Shang, Y. M. Wulia, and X. F. Wang, "Protective effects of Cistanche glycosides on ultrastructure of sensitive organs of ${ }^{60} \mathrm{Co}$ irradiated mice," Northwest Pharmaceutical Journal, vol. 16, no. 2, pp. 66-67, 2001.

[88] G. H. Wu, J. Yu, R. H. Zhou, Q. Lin, J. Wang, and S. D. Piao, "Effect of $\beta$-aescine sodium with mannito on radiation-induced brain edema," Journal of Practical Diagnosis and Therapy, vol. 22, no. 2, pp. 110-111, 2008.

[89] Y. R. Li, J. Tan, J. Wang, S. L. Xu, and J. Guo, "Effect of radix hedysari capsule on 60Co 7 after radiation in lung and brain of rats, the serum SOD activity and MDA content," Shanxi University of Traditional Chinese Medicine, vol. 31, no. 3, pp. 363364, 2010.

[90] Y. R. Li, J. Tan, J. Wang et al., "The radiation-protective effect of radix hedysari capsules on mice," Pharmaceutical Journal of Chinese People's Libration Army, vol. 26, no. 5, pp. 400-402, 2010.

[91] Y. H. Xie, J. Guo, Q. Yang, S. W. Wang, and Y. R. Li, “The protective effect of hongqi capsule on low-dose radiation injured mice," Progress in Modern Biomedicine, vol. 15, no. 18, pp. 3452-3457, 2015.

[92] L. Gan, Z. H. Wang, H. Zhang, C. J. Ma, and G. Li, "Protective effects of hydroxy safflower yellow A on radiation-induced brain injury induced by carbon ion beam irradiation," Nuclear Techniques, vol. 35, no. 8, pp. 624-629, 2012. 
[93] L. Gan, Z. H. Wang, H. Zhang et al., "Protective effects of shikonin on brain injury induced by carbon ion beam irradiation in mice," Biomedical and Environmental Sciences, vol. 28, no. 2, pp. 148-151, 2015.

[94] R. W. Stackman, F. Eckenstein, B. Frei, D. Kulhanek, J. Nowlin, and J. F. Quinn, "Prevention of age-related spatial memory deficits in a transgenic mouse model of Alzheimer's disease by chronic Ginkgo biloba treatment," Experimental Neurology, vol. 184, no. 1, pp. 510-520, 2003.

[95] M. C. J. M. van Dongen, E. van Rossum, A. G. H. Kessels, H. J. G. Sielhorst, and P. G. Knipschild, "The efficacy of ginkgo for elderly people with dementia and age-associated memory impairment: New results of a randomized clinical trial," Journal of the American Geriatrics Society, vol. 48, no. 10, pp. 1183-1194, 2000.

[96] Y. Q. Ding, Y. Wang, and X. J. Chen, "Effect of Ginkgo flavonoid on acute radiation-induced hippocampal injury in rats," Jiangsu Medical Journal, vol. 39, no. 20, pp. 2379-2382, 2013.

[97] A. M. Sun, C. G. Li, S. M. Lin, Y. Q. Zhang, and Q. Xia, “The importance of regulatory role of panaxoside Rg1 in Cdk5 on hippocampal neuron radioactive damage protection," Journal of Chinese Physician, vol. 16, no. 5, pp. 584-587, 2014.

[98] S. Hu, R. Peng, C. Wang et al., "Neuroprotective effects of dietary supplement Kang-fu-ling against high-power microwave through antioxidant action," Food \& Function Journal, vol. 5, no. 9, pp. 2243-2251, 2014.

[99] J. Zhang, F. Tong, Q. Cai et al., "Shenqi Fuzheng Injection attenuates irradiation-induced brain injury in mice via inhibition of the NF- $\kappa$ B signaling pathway and microglial activation," Acta Pharmacologica Sinica, vol. 36, no. 11, pp. 1288-1299, 2015.

[100] G. Y. Zhu, W. M. Cai, and K. Liang, "Preliminary study on prevention and treatment of radiation brain injury in acute stage by 978-1," Chinese Journal of Radiological Medicine and Protection, vol. 20, no. 3, p. 163, 2000.

[101] G. Y. Zhu, K. Liang, and W. M. Cai, "Mechanism and effect of Chinese medicine destagnation and renal invigoration on radiation brain injury in mice," Chinese Journal of Radiation Oncology, vol. 10, no. 1, pp. 38-41, 2001.

[102] B. Yang, B. X. Ren, and F. R. Tang, "Prenatal irradiationinduced brain neuropathology and cognitive impairment," Brain \& Development, vol. 39, no. 1, pp. 10-22, 2017.

[103] J. P. Wang, X. J. Zhang, X. W. Yu, G. H. Xing, Y. R. Sun, and W. Rong, "Effects of Beta-asarone on hippocampal neurons of depression rat model," Journal of Guangzhou University of TCM, vol. 31, no. 6, pp. 924-927, 2014.

[104] G. Z. Hu, R. Q. Nie, Y. S. Xiao, J. Zhang, Z. Wen, and D. F. $\mathrm{Wu}$, "Influence of polygonatum polysaccharide on apotosis of primary cultured neonate rat cerebral cortical neurons caused by hypoxia," Pharmacology and Clinics of Chinese Materia Medica, vol. 21, no. 4, pp. 37-39, 2005.

[105] X. Y. Huang, Y. Wang, X. M. Zhang, J. C. Zhao, and X. F. Zhu, "Effects of polygalaceae ssponins on neural stem cell differentiation into neurons," Neural Injury and Functional Reconstruction, vol. 6, no. 2, pp. 90-93, 2011.

[106] S. W. Wang, B. X. Ren, F. Qian et al., "Radioprotective effect of epimedium on neurogenesis and cognitionafter acute radiation exposure," Neuroscience Research, vol. S0168-0102, no. 18, pp. 30380-30388, 2018.

[107] F. Jin, C. Chen, H. Jin et al., "Protective effects of icariin on spatial learning and memory in rats with AB25-35 induced
Alzheimer's disease via inhibiting TNF-a, IL-6 and caspase3 expression," Chinese Journal of New Drugs and Clinical Remedies, vol. 32, no. 10, pp. 833-837, 2013.

[108] W. Q. Zhou, J. Y. Deng, M. G. Bi, and G. H. Du, "Correlation between effect of icariin on learning and memory and level of estradiol in serum in senescence accelerated mouse prone 8," Chinese Journal of Experimental Traditional Medical Formulae, vol. 15, no. 9, p. 341, 2009.

[109] X. F. Zhang, "Toxic and side effects of Chinese medicine and preventive measures," Chinese Medicine Modern Distance Education of China, vol. 10, no. 11, pp. 65-66, 2012.

[110] M. Zhou, Y. Hong, X. Lin, L. Shen, and Y. Feng, "Recent pharmaceutical evidence on the compatibility rationality of traditional Chinese medicine," Journal of Ethnopharmacology, vol. 206, pp. 363-375, 2017. 


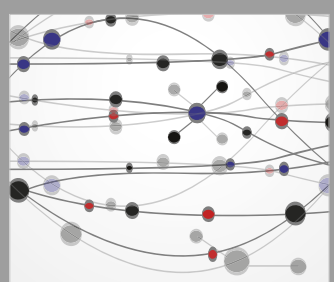

The Scientific World Journal
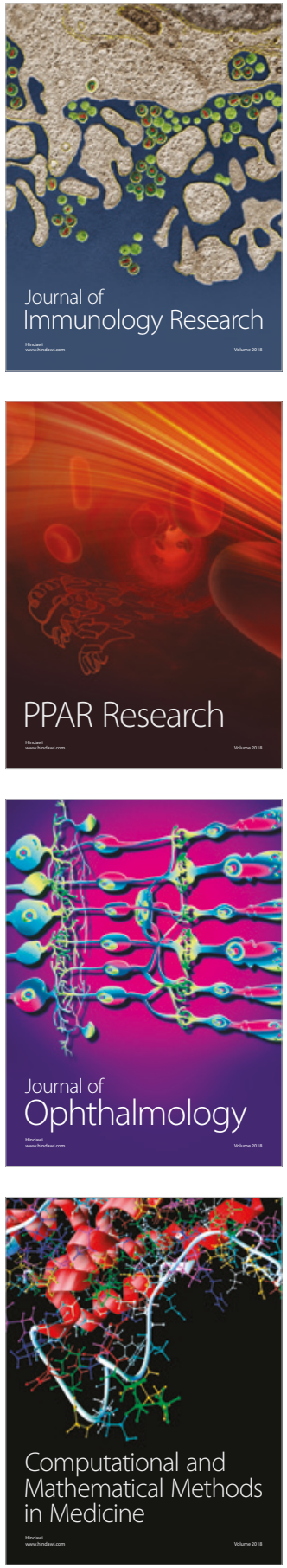

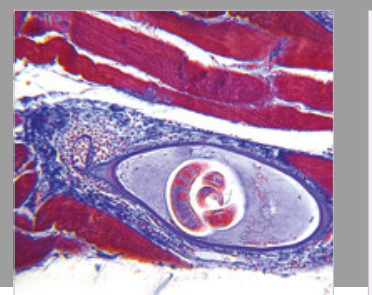

Gastroenterology Research and Practice

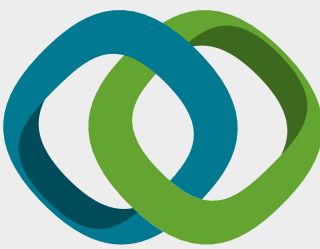

\section{Hindawi}

Submit your manuscripts at

www.hindawi.com
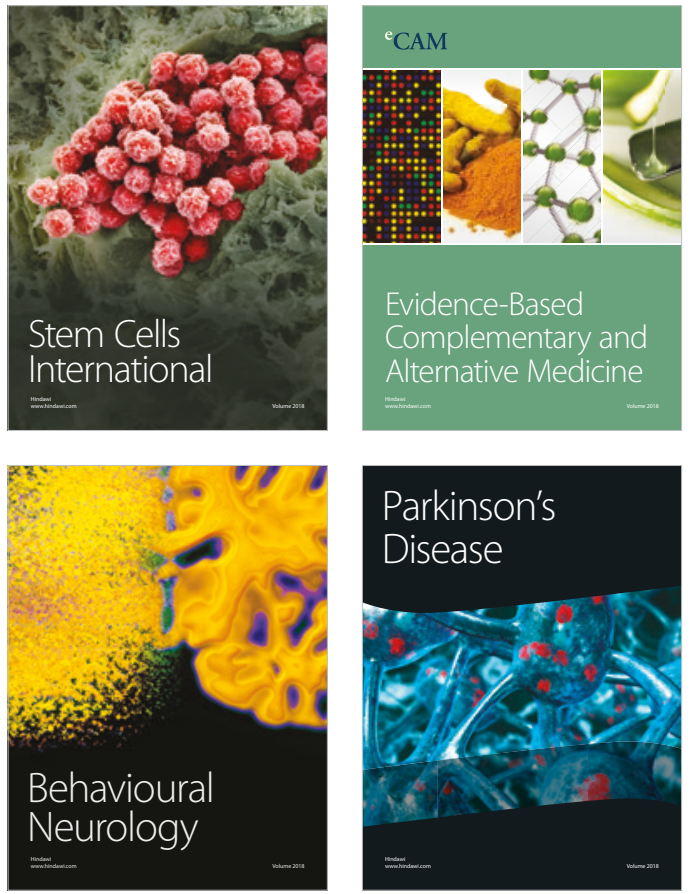

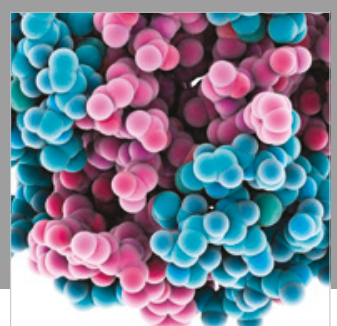

ournal of

Diabetes Research

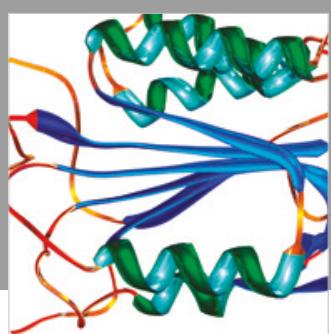

Disease Markers
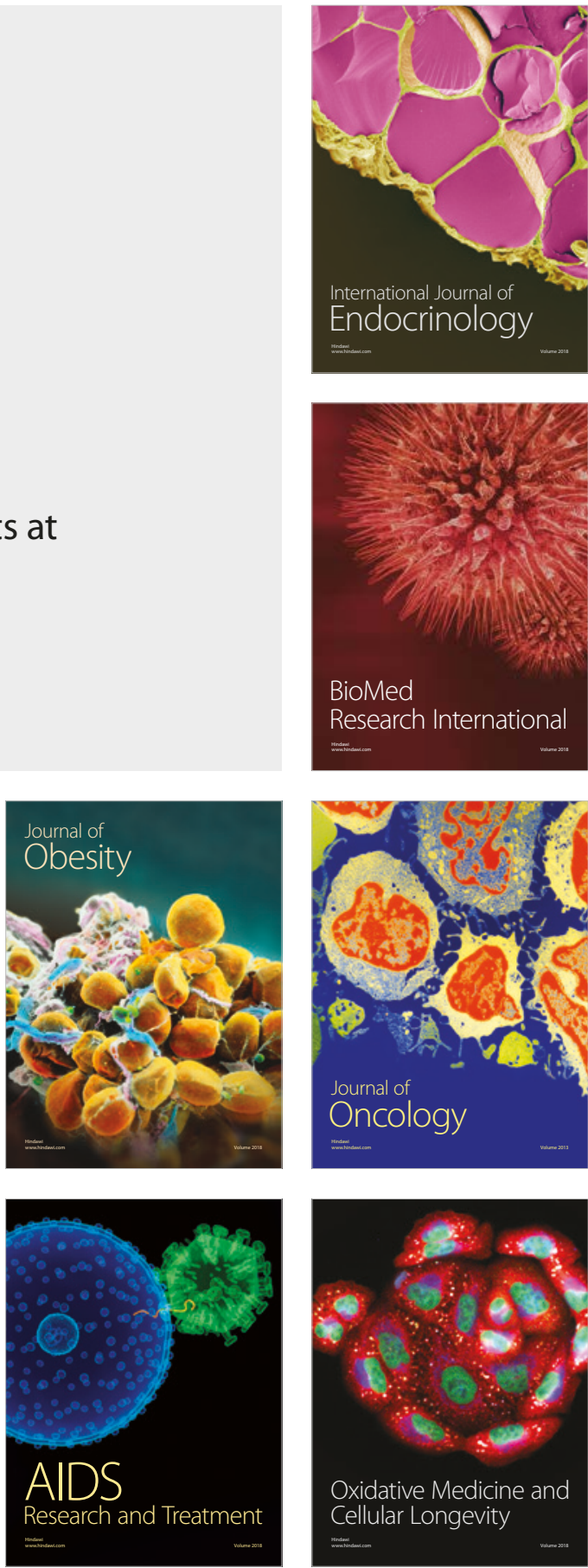